\title{
APPROXIMATE DETERMINATION OF RADIATION PROPERTIES IN THE ARC PLASMAS WITH ADMIXTURES OF COPPER VAPOURS
}

\author{
N. Bogatyreva ${ }^{a, *}$, M. Bartlovía ${ }^{a}$, V. Aubrecht ${ }^{b}$, P. Kloc ${ }^{b}$ \\ a Department of Physics, Faculty of Electrical Engineering and Communication, Brno University of Technology, \\ Technická 3058/10, 61600 Brno, Czech Republic \\ ${ }^{b}$ Department of Power Electrical and Electronic Engineering, Faculty of Electrical Engineering and \\ Communication, Brno University of Technology, Technická 3058/12, 61600 Brno, Czech Republic \\ * bogatyreva@feec.vutbr.cz
}

\begin{abstract}
The aim of this paper is to evaluate radiation properties of air arc plasmas with various admixtures of copper vapours. The first order of the method of spherical harmonics $\left(P_{1}\right.$-approximation) has been used as the way to solve the equation of radiation transfer. Calculations of the absorption coefficients for a thermal plasma have been performed as a function of the temperature and the frequency. The frequency variable in the equation of transfer was handled by means of multigroup method. Methods for prediction of the average absorption coefficients were described and compared in detail. The net emission coefficients have been determined for comparison.
\end{abstract}

Keywords: arc plasma, radiation transfer, absorption properties, admixtures of copper vapours.

\section{Introduction}

This paper presents the methods for research and prediction of the radiation properties in electric arc plasmas. Radiation transport of energy plays an important role in many plasma processing devices. Electric arc plasmas are utilized in a number of industrial applications. The electric arc parameters depend on the purity of gas in the chamber and the controllability of other additives in plasma. In many cases concentration of different species in the plasma is not possible to measure or measurement of different parameters will be difficult for technical realization. Then, modeling of the plasma spectrum is highly important for investigation of electric arc.

\section{Energy transfer}

Mathematical modeling is necessary to determine of radiation flux and its divergence. The solution of the equation of radiation transfer makes possible to estimate these parameters. The complete stationary equation of radiation transfer for an absorbing and emitting medium is

$$
\boldsymbol{\Omega} \cdot \operatorname{grad}\left(I_{\nu}\right)=\kappa_{\nu}\left[B(\nu)-I_{\nu}\right],
$$

where $\boldsymbol{\Omega}$ is the unit direction vector, $I_{\nu}$ is the radiation intensity, $\kappa_{\nu}$ is the absorption coefficient, $B(\nu)$ is the Planck's spectral radiation intensity for equilibrium radiation, $\nu$ is the frequency [1].

The spectral intensity depends in general upon independent variables $(\mathbf{r}, \nu, \boldsymbol{\Omega})$. One must approximate the equation of transfer, either analytically or numerically, in order to obtain a solution. Due to the non-linearity of the equation describing the radiation field and the strong dependence of input parameters on the radiation frequency, various approximate methods are used. One of them is the method of spherical harmonics $P_{N}$-approximation.

The $P_{1}$-approximation consists of expanding the radiation intensity in spherical harmonics and including only the first order terms [2]. Under this assumption, the spectral equation of radiation transfer leads to a simple elliptic equation for radiation density $U_{\nu}$

$$
\begin{array}{r}
-\operatorname{div}\left\{\frac{c}{3 \kappa_{\nu}} \operatorname{grad}\left[U_{\nu}(\mathbf{r}, \nu)\right]\right\} \\
+\kappa_{\nu} c U_{\nu}(\mathbf{r}, \nu) \\
=\kappa_{\nu} 4 \pi B(\nu),
\end{array}
$$

where $c$ is the speed of light, $\mathbf{r}$ is the position vector fixing the location of a point in space.

\section{Absorption coefficients}

Radiation in arc plasma depends on concentrations of all chemical species present in the plasma. Equilibrium compositions of plasma can be computed by means of several methods. In our case, plasma compositions are calculated by using the Tmdgas computer code, which is part of the database system TheCoufal $[3,4]$. Our calculations take into account continuum and line radiation of atomic and ionic species and photoabsorption of diatomic molecules. It enables to obtain an approximate solution of required accuracy. One of the options considered is clean air without $\mathrm{CO}_{2}$. We assume that the plasma containes some elements $\mathrm{O}_{2}, \mathrm{~N}_{2}, \mathrm{Ar}, \mathrm{Cu}, \mathrm{e}^{-}$and also ions of $\mathrm{O}, \mathrm{N}$, $\mathrm{Ar}, \mathrm{Cu}$.

Comparison of temperature dependences of species concentration in the arc plasma for various admixtures of copper vapours is presented in Fig. 1. Compositions were calculated for admixtures of $5 \%, 50 \%, 95 \% \mathrm{Cu}$ and pure air at pressure $0.1 \mathrm{MPa}$. 

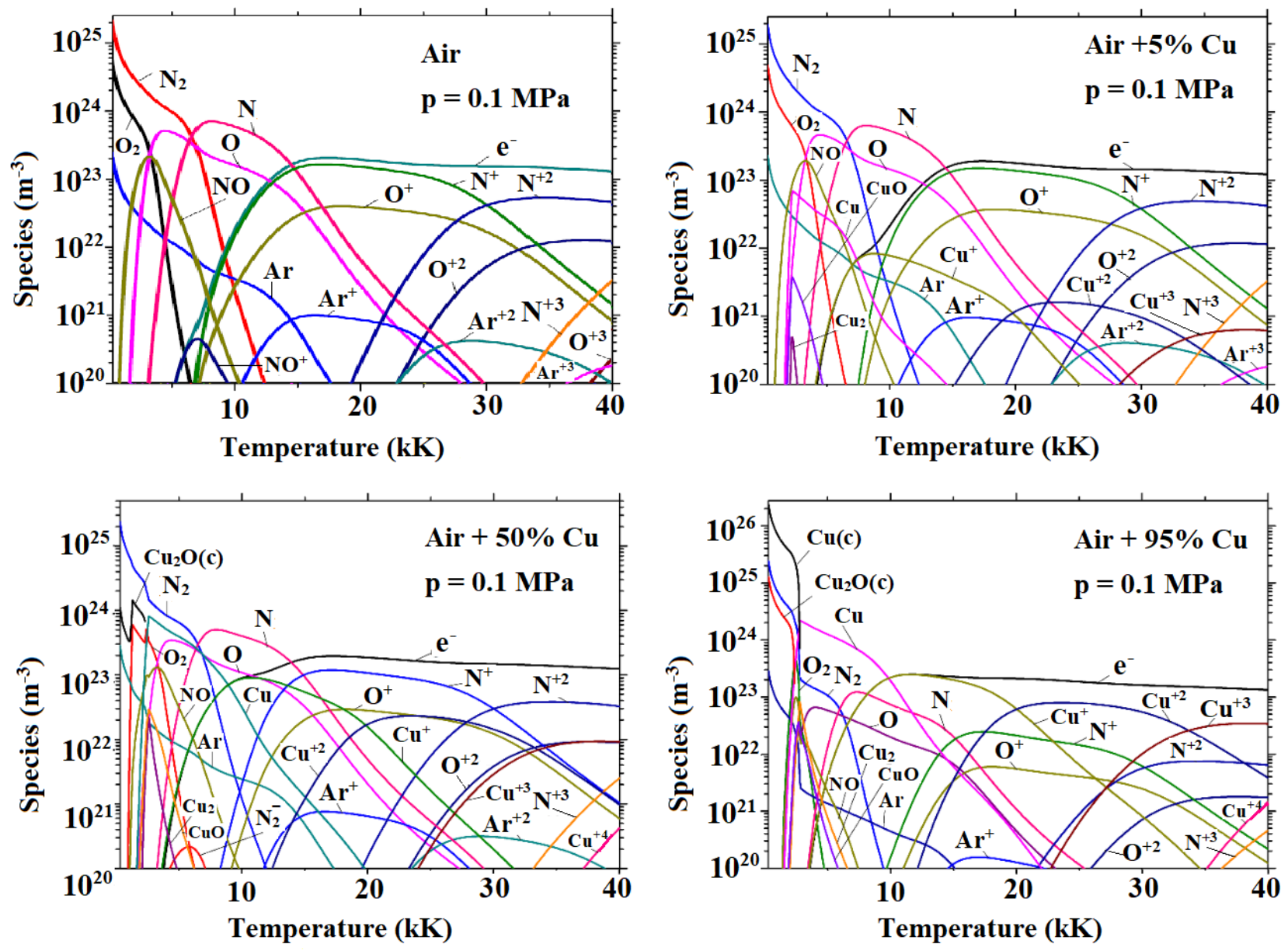

Figure 1. Concentration of different species in the thermal plasma as a function of temperature.

For atomic and ionic species, the absorption coefficients $\kappa_{\nu}$ were calculated. Both line and continuous radiation were considered in the calculation.

The total coefficient of absorption is given by the linear sum

$$
\kappa_{\nu}(\nu, T, p)=\kappa_{\nu}^{\mathrm{ff}}+\kappa_{\nu}^{\mathrm{bf}}+\kappa_{\nu}^{\mathrm{bb}} .
$$

The indexes "ff, bf, bb" denote free-free transitions (bremsstrahlung), bound-free transitions (photorecombination) and bound-bound transitions (discrete radiation), respectively.

The absorption coefficients of air plasma were calculated for three concentrations of copper (5\%, 50\%, $95 \%)$ at a pressure of $0.1 \mathrm{MPa}$. The plasma temperature was considered in the range of $(300,35000) \mathrm{K}$. The frequency interval for calculations was (0.01 to 10) $\cdot 10^{15} \mathrm{~Hz}$ with increment of $10^{12} \mathrm{~Hz}$.

Evaluated total spectral absorption coefficients of air arc plasmas with various admixtures of copper vapours at temperature $15000 \mathrm{~K}$ are shown in Fig. 2. This spectrum is a complex of discrete and continuous spectra. Both dependences of the absorption coefficients on the radiation frequency and the concentrations of copper vapours can be seen in Fig. 2 . The concentration of admixtures has an effect on the magnitude of the absorption coefficients. The greater influence is found in the first half of the frequency interval considered. The increase of the admixtures of copper vapours lead to an increase of the number of spectral lines.

\section{Mean absorption coefficients}

The simplification of the frequency dependence imposes a cutting of the spectrum in several frequency groups, in which the absorption coefficient is supposed to be constant with certain average value. We have calculated Planck $\left(\kappa_{\mathrm{P}}\right)$ and Roseland $\left(\kappa_{\mathrm{R}}\right)$ means for cutting the spectrum into 5 frequency groups. Fig. 3 shows the mean Planck and Rosseland absorption coefficients as a function of the temperature for air plasma with admixtures of $5 \% \mathrm{Cu}$ and $95 \% \mathrm{Cu}$ at the pressure of $0.1 \mathrm{MPa}$. Admixtures of copper vapours increase particularly the Planck absorption means since the Planck means stronger dependent upon the spectral lines.

The Planck mean is appropriate in the case of optically thin and emission dominated systems. It can be 

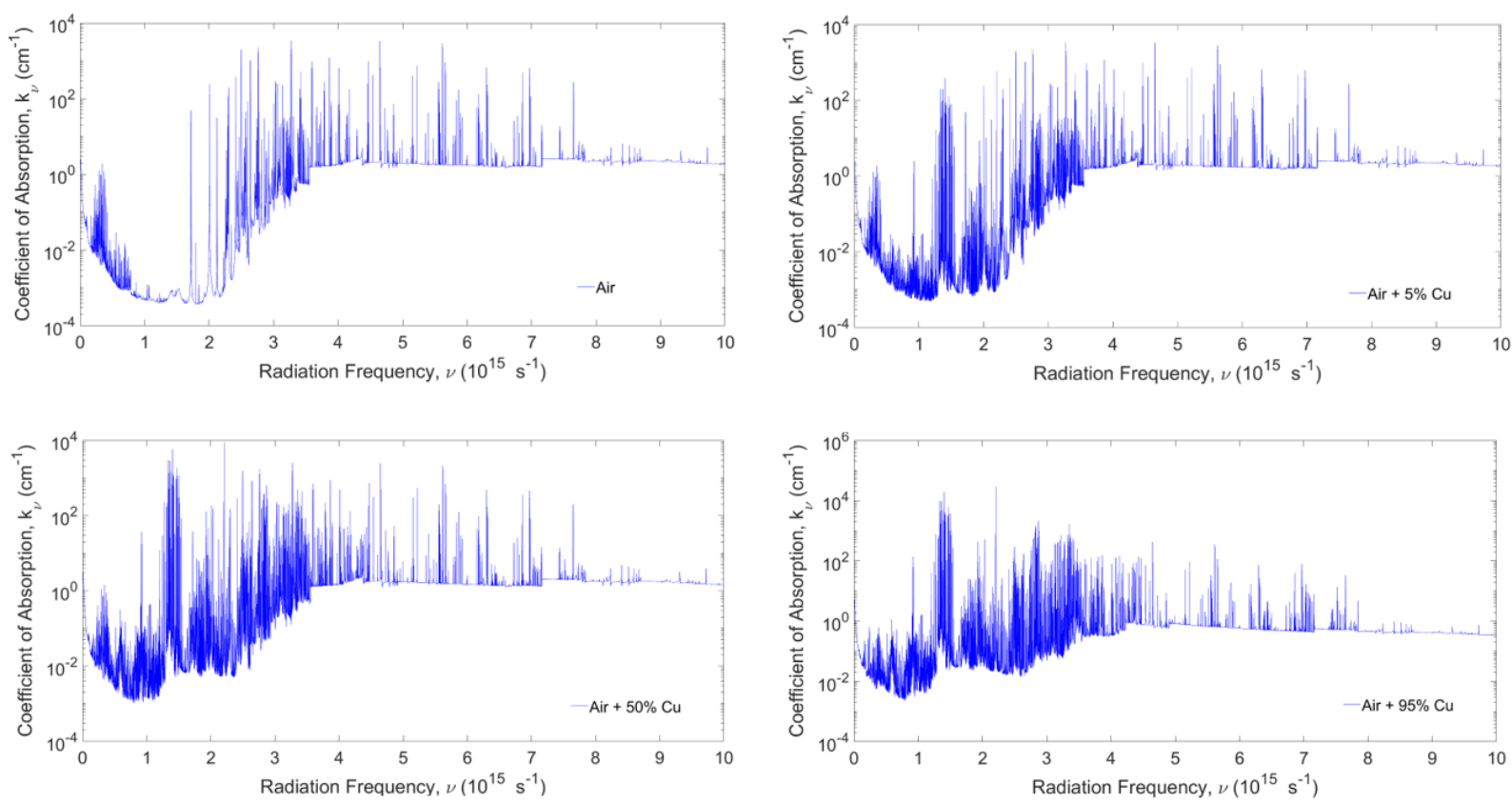

Figure 2. Comparison of absorption coefficients in thermal plasma for different concentrations of copper.

expressed as

$$
\overline{\kappa_{\mathrm{P}}}=\frac{\int_{\nu_{k-1}}^{\nu_{k}} \kappa_{\nu} B(\nu, T) \mathrm{d} \nu}{\int_{\nu_{k-1}}^{\nu_{k}} B(\nu, T) \mathrm{d} \nu} .
$$

Here, radiation intensity is given by the Planck function

$$
B(\nu, T)=\frac{2 h \nu^{3}}{c^{2}\left[\exp \left(\frac{h \nu}{k_{B} T}\right)-1\right]},
$$

where $h$ is the Planck constant, $k_{B}$ is the Boltzmann constant, $T$ is the temperature.

The Rosseland mean, also called mean free path of radiation is appropriate when the system approaches equilibrium (almost all radiation is reabsorbed). It has the form

$$
\overline{\kappa_{\mathrm{R}}^{-1}}=\frac{\int_{\nu_{k-1}}^{\nu_{k}} \kappa_{\nu}^{-1} \frac{\mathrm{d} B(\nu, T)}{\mathrm{d} T} \mathrm{~d} \nu}{\int_{\nu_{k-1}}^{\nu_{k}} \frac{\mathrm{d} B(\nu, T)}{\mathrm{d} T} \mathrm{~d} \nu} .
$$

The use of Rosseland and Planck mean absorption coefficients is only strictly appropriate in limiting circumstances. In the multigroup method, the splitting of the whole frequency interval has to be made according to frequency dependence of the absorption coefficients. For frequency groups with low values of absorption coefficients, the use of Planck mean is appropriate; for groups with high values of absorption coefficients, the Rosseland mean is more suitable. In our case, for groups 1-4 the Planck mean gives better results, in the group 5 the Rosseland mean is more appropriate.
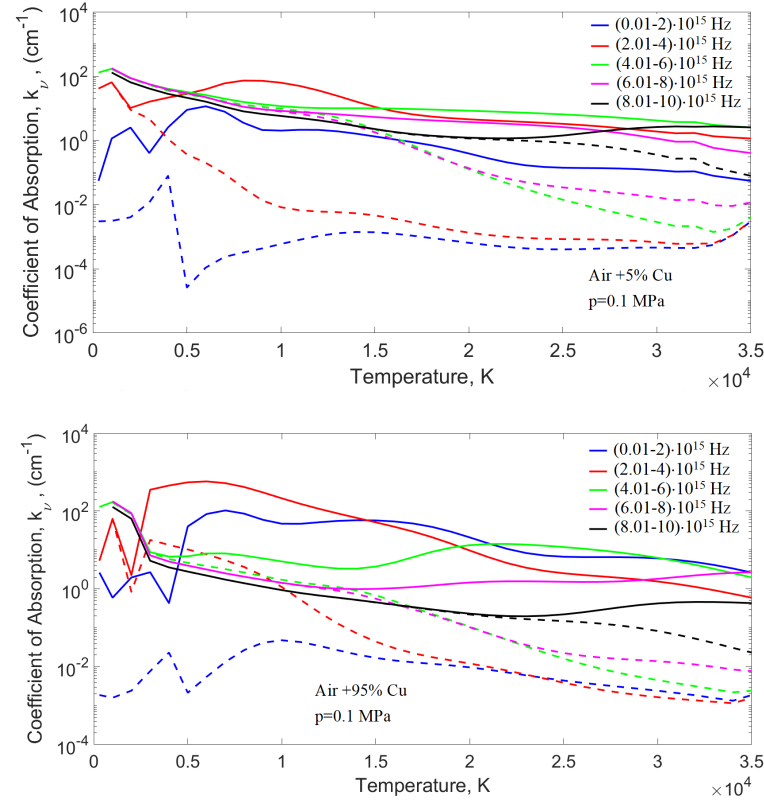

Figure 3. Planck (full lines) and Rosseland (dotted lines) means absorption coefficients for various frequency groups as a function of temperature.

\section{Isothermal plasma cylinder}

In the case of a cylindrically symmetrical plasma, the multigroup equation (2) depends only on one variable - the radial range $r$. Due to the fact that functions $\bar{\kappa}_{n}$ (mean absorption coefficient for any frequency interval) and $B_{n}$ (Planck function for any frequency interval) are dependent on the arc temperature and it decreases from the axis to the edge they are usually regarded as radial range functions. If we assume (to simplify the task) that the plasma is isothermal, i.e. 

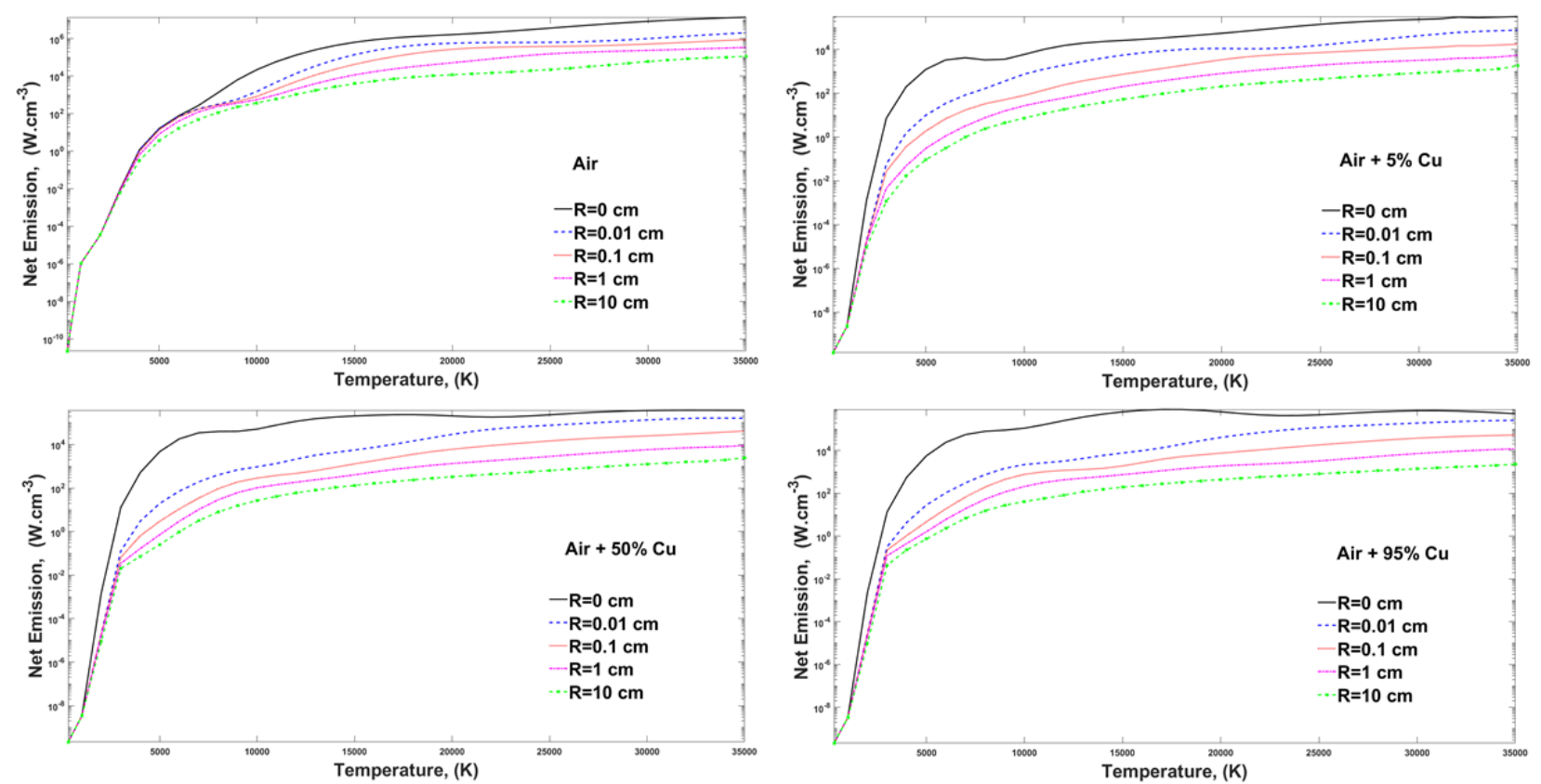

Figure 4. Net emission coefficient of isothermal plasma cylinder as a function of temperature for various thicknesses of the plasma.

the arc temperature remains constant, functions $\bar{\kappa}_{n}$ and $B_{n}$ also remain constant for the current solution of equation (2).

If no radiation enters from outside, the group net emission over the volume of the arc is

$$
\begin{array}{r}
\left(w_{\mathrm{avg}}\right)_{n}=\frac{2 \pi}{\pi R^{2}} \int_{0}^{R} r \operatorname{div} \mathbf{W}_{n}(r) \mathrm{d} r \\
=\frac{8 \pi B_{n} J_{1}\left(\sqrt{3} \bar{\kappa}_{n} R\right)}{R\left[2 J_{1}\left(\sqrt{3} \bar{\kappa}_{n} R\right)+\sqrt{3} J_{0}\left(\sqrt{3} \bar{\kappa}_{n} R\right)\right]},
\end{array}
$$

where $J_{1}(x)$ and $J_{0}(x)$ are the modified Bessel functions, $R$ is the arc radius, and $\operatorname{div} \mathbf{W}_{n}(r)$ is the group radiation flux.

The sum over all frequency groups gives the net emission of radiation

$$
\operatorname{div} \mathbf{W}_{R}=w_{\mathrm{avg}}=\sum_{n}\left(w_{\mathrm{avg}}\right)_{n}=4 \pi \varepsilon_{N},
$$

where $\varepsilon_{N}$ is the net emission coefficient.

The isothermal net emission coefficient corresponds to the fraction of the total power per unit volume and unit solid angle irradiated into a volume surrounding the axis of the arc plasma and escaping from the arc column after crossing a thickness $R$ of the isothermal plasma.

The net emission coefficient for the isothermal plasma cylinder with radius $R$ is given by [5]

$$
\varepsilon_{N}=\int_{0}^{\infty} B(\nu) \kappa_{\nu} \exp \left(-\kappa_{\nu} R\right) \mathrm{d} \nu
$$

and can be calculated with a direct integration over the real spectrum.
Comparison of the net emission coefficients for five different radii of the plasma cylinder $(0 \mathrm{~cm} ; 0.01 \mathrm{~cm}$; $0.1 \mathrm{~cm} ; 1 \mathrm{~cm} ; 10 \mathrm{~cm}$ ) at a pressure of $0.1 \mathrm{MPa}$ is presented in the Fig. 4. The zero radius corresponds to the case omitting self-absorption. Besides the temperature variation, the plasma thickness has great influence on the radiation emission.

\section{Results}

The radiation properties in the arc plasmas were determinated by using the approximate method mentioned above. Plasma compositions with admixtures of $5 \%$, $50 \%, 95 \%$ copper vapours were prepared at a pressure of $0.1 \mathrm{MPa}$ in the temperature interval $(300,35000) \mathrm{K}$. Absorption coefficients for a plasma with these admixtures of $\mathrm{Cu}$ were calculated and the spectra were plotted in a wide frequency range. Mean absorption coefficients were obtained by employing Planck and Rosseland means. Then, net emission coefficients were computed for different radii of the isothermal plasma cylinder. Based on the determined radiation parameters it is possible to analyze the influence for different admixtures of copper vapours $(5 \%, 50 \%, 95 \%)$ in the arc plasmas. Results of our work will be useful for practical purposes, for example in the construction of circuit breakers.

Many authors have calculated the parameters for air plasma with admixtures of copper vapours. Most of them perform computations for $1 \%, 10 \%$ or $100 \%$ of $\mathrm{Cu}$. Our calculations were carried out for other concentrations. Results of our work were compared with values of net emission coefficients of Cressault [6] and Aubrecht [7]. Our method allows us to get a 

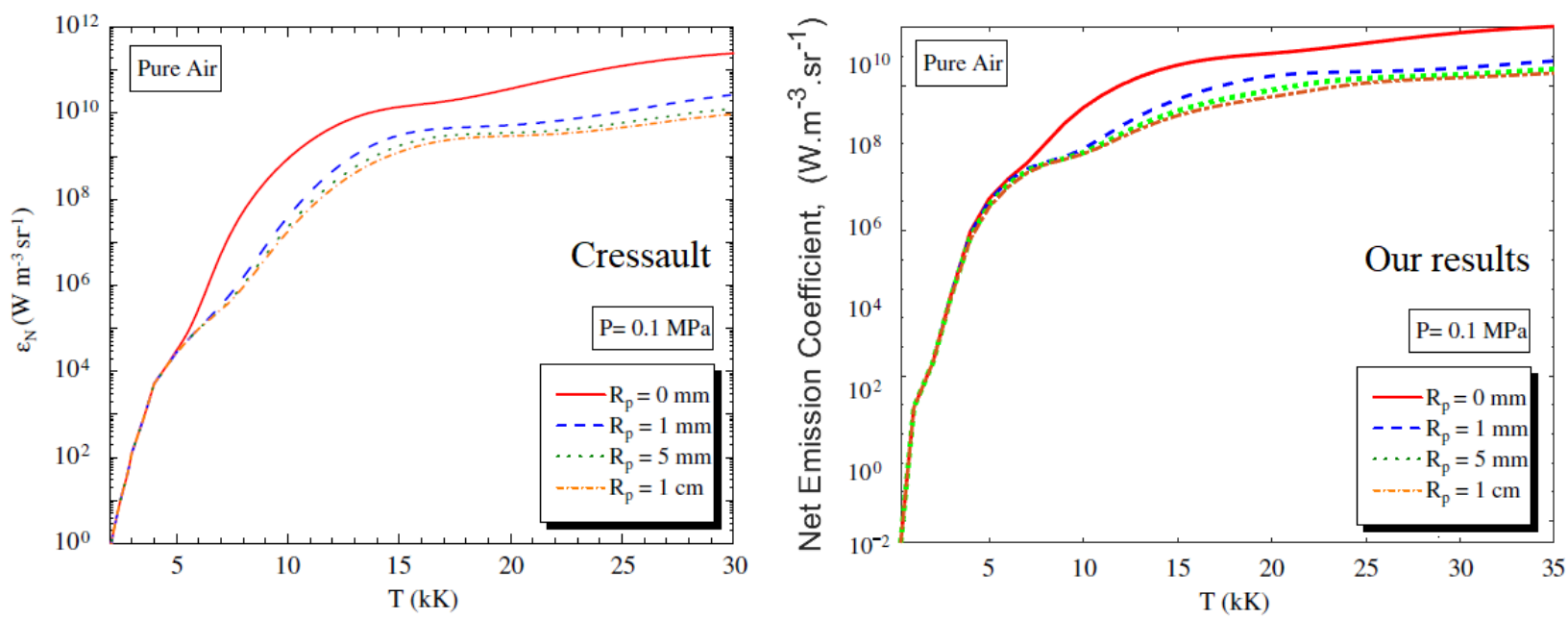

Figure 5. Comparison of net emission coefficient in air plasma.

satisfactory agreement with the calculations of other authors (Fig. 5, Fig. 6). Discrepancies between the results can be explained by the different approximate methods of calculation.

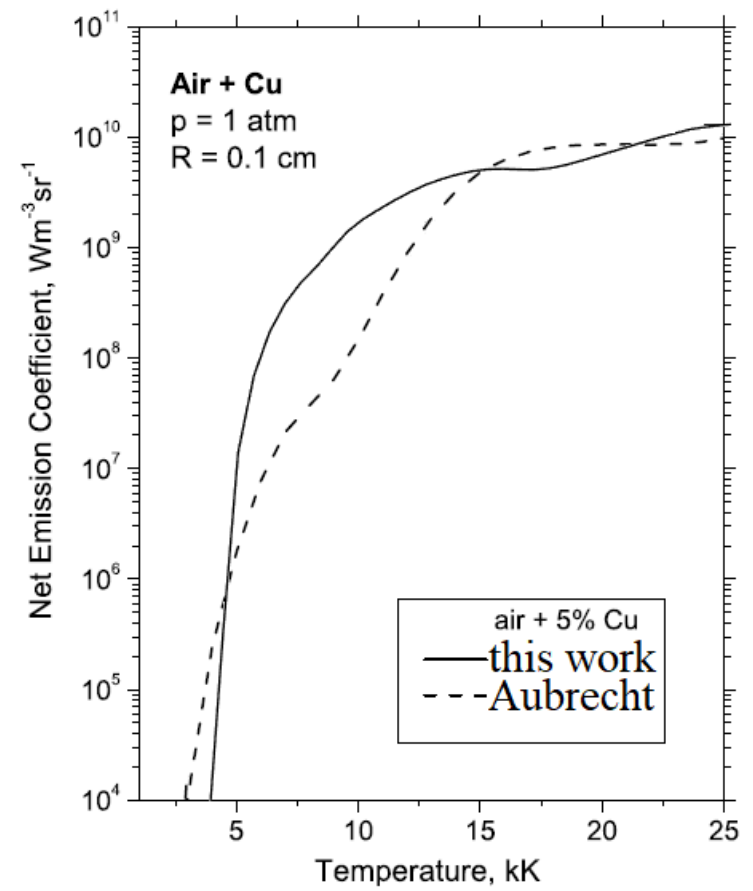

Figure 6. Net emission coefficient in air plasma with $5 \% \mathrm{Cu}$.

\section{Conclusions}

The Rosseland and Planck mean absorption coefficients can differ by several orders of magnitude. Discrete radiation influences significantly the values of Planck means; on the other hand, Rosseland means ignore the role of lines mainly at lower frequencies. Admixture of $\mathrm{Cu}$ vapour in air arc plasma contributes to the increase of the net emission. The contribution depends on the $\mathrm{Cu}$ vapour concentrations. The choice of mixtures was guided by the amount of evaporated electrode material in self-blast circuit breakers with copper electrodes.

\section{Acknowledgements}

This research work was carried out in the Centre for Research and Utilization of Renewable Energy (CVVOZE). The authors gratefully acknowledge financial support from the Ministry of Education, Youth and Sports of the Czech Republic under NPU I programme (project No. LO1210 Energy for Sustainable Development) and OP VVV Programme (project No. CZ. 02.1.01/0.0/0.0/16o13/0001638 CVVOZE Power Laboratories-Modernization of Research Infrastructure).

\section{References}

[1] M. I. Boulos, P. Fouchais, and E. Pfender. Thermal plasmas: fundamentals and applications. 1. Plenum Press, New York, 1994.

[2] B. N. Chetverushkin. Matematicheskoe modelirovanie zadach dinamiki izluchajuschego gaza. Nauka, Moskva, 1985.

[3] O. Coufal. Database system of thermodynamic properties of individual substances at high temperatures. J.Phys.D: Appl.Phys., 38(8):1265-1274, 2005. doi: 10.1088/0022-3727/38/8/026.

[4] O. Coufal. Tmdgas. arXiv:http://www.feec.vutbr.cz/ coufal.

[5] J. Lowke. Prediction of arc temperature profiles using approximate emission coefficients for radiation losses. $J$. Quant. Spectroscop. Radiat. Transfer, 14(2):111-122, 1974. doi : 10.1016/0022-4073(74)90004-1.

[6] Y. Cressault, R. Hannachi, P. Teulet, A. Gleizes, J.-P. Gonnet, and J.-Y. Battandier. Influence of metallic vapours on the properties of air thermal plasmas. Plasma Sources Sci. Technol., 17(3):35016, 2008. doi:10.1088/0963-0252/17/3/035016.

[7] V. Aubrecht, M. Bartlová, and O. Coufal. Radiative emission from air thermal plasmas with vapour of $\mathrm{Cu}$ or W. J. Phys. D: Appl. Phys., 43(43):434007, 2010. doi : $10.1088 / 0022-3727 / 43 / 43 / 434007$. 